\title{
Improvement of thermal properties of poly (L-lactic acid) by blending with zinc lactate
}

\author{
Yan-Hua Cai ${ }^{1,2^{*}}$, Li-Ping Ren ${ }^{1,2}$ and Ying Tang ${ }^{1,2}$ \\ ${ }^{I}$ Chongqing Key Laboratory of Environmental Materials and Remediation Technologies, Chongqing University of Arts and Sciences, \\ Yongchuan, Chongqing-402160, P.R. China. \\ ${ }^{2}$ School of Materials and Chemical Engineering, Chongqing University of Arts and Sciences, Yongchuan, Chongqing-402160, P.R. China.
}

\begin{abstract}
Zinc lactate (RSX) was synthesised from zinc acetate and L-lactic acid, and the structure was characterised using X-ray diffraction (XRD). Thermal decomposition showed that the decomposition temperature of RSX is higher than the processing temperature of composites based on poly (L-lactic acid) (PLLA). Single factor experiments certified that the optimum reaction conditions are a reaction temperature of $80^{\circ} \mathrm{C}$, and a reaction time of 4 hours. Addition of RSX could significantly decrease the half-time of overall PLLA crystallisation. Compared to pure PLLA, the half-time of PLLA with $7 \%$ RSX decreased from 2359.91 seconds to 152.68 seconds at $115{ }^{\circ} \mathrm{C}$. The kinetics of isothermal crystallisation of PLLA/RSX composites can be described using the Avrami equation.
\end{abstract}

Keywords: Crystallisation kinetics, isothermal crystallisation, poly (L-lactic acid), thermal decomposition, zinc lactate.

\section{INTRODUCTION}

Zinc lactate (RSX) is an important nutrient used to overcome zinc deficiency in food. It is often used in cosmetics and toothpaste (Cao et al., 2002). Continuous studies have been made on the crystallisation and dissolution behaviour of RSX (Zhang et al., 2010; 2012). RSX will also find applications in many fields. Effects of stirring and ultrasonic waves on the crystallisation of RSX have already been investigated (Cao et al., 2002). The results show that stirring could improve dissolution of crystallized RSX, and ultrasonic waves do not influence the crystallisation process.

Poly (L-lactic acid) (PLLA), is an important biodegradable polymer material widely investigated by researchers and has been used in many ways such as in packaging (Longano et al., 2012), automobile industry (Jo et al., 2012) and drug delivery (Campardelli et al., 2014). To widen the application of PLLA in food packaging, PLLA/montmorillonite nanocomposites have been fabricated by melt processing. The results show that PLLA/montmorillonite composite films could exhibit enhancement in oxygen and water vapour barrier properties (Kotiyar et al., 2011).

A few disadvantages occur to restrict the application of PLLA, such as slow crystallisation rate, low degree of crystallisation and poor heat resistance (Cai et al., 2014). A wider range of applications would be possible if the thermal properties of PLLA could be improved. Usually, blending with some functional additives is often used to improve the thermal properties of PLLA. For example, addition of ethylene bis-12-hydroxystearamide significantly improves the crystallisation rate and crystallinity of PLA. Compared to pure PLA, only the half-time of PLA with $1 \%$ ethylene bis-12hydroxystearamide at $105{ }^{\circ} \mathrm{C}$ decreased from 18.8 minutes to 2.8 minutes, and the crystallinity of PLA with $1 \%$ ethylene bis-12-hydroxystearamide is $35 \%$ after 5 minute heat treatment (Tang et al., 2012).

In this study, RSX was synthesised to add to PLLA matrix using melting blending technology. The thermal properties of PLLA/RSX composites were investigated in detail to elucidate the role of RSX in enhancement of thermal properties of PLLA/RSX. This study may also help researchers to obtain more functional additives for PLLA. 


\section{METHODS AND MATERIALS}

\section{Materials}

Poly(L-lactic acid) (2002D) was purchased from Nature Works L.L.C., USA. Zinc acetate and L-lactic acid of analytical grade were purchased from Chengdu Kelong Chemical Reagents Company (Sichuan Province, China), and ethanol of analytical grade was purchased from Tianjin Kemiou Chemical Reagents Company (Tianjin, China).

\section{Synthesis of RSX}

Six grams of zinc acetate was dissolved in $180 \mathrm{~mL}$ of ethanol and $5 \mathrm{~mL}$ of water. The mixture was heated at $80^{\circ} \mathrm{C}$ for $3 \mathrm{~h}$ while stirring. Then $2.5 \mathrm{~mL}$ of L-lactic acid was added slowly into the mixture and kept at $80^{\circ} \mathrm{C}$ for $4 \mathrm{~h}$ while stirring. The reaction mixture was cooled to room temperature and filtered. The obtained crude product was washed with ethanol and water, and the resulting product was dried under vacuum at $45^{\circ} \mathrm{C}$.

\section{Preparation of PLLA/RSX composites}

The preparation process of PLLA/RSX composite was similar to the process of preparing other PLLA materials as reported previously (Cai, 2013).

\section{Characterisation}

X-ray diffraction (XRD): XRD analyses of RSX were performed on a X-ray diffractometer (D/MAX2550, Rigaku, Japan) using $\mathrm{Cu} K_{\alpha}$ radiation (wavelength, 1.54 $\AA$ ) in the range of $2 \theta=5-80^{\circ}$ with a scanning rate of $2 \%$ min.

Thermogravimetric analysis (TGA): Thermogravimetric analysis of RSX was performed using a thermal analysis Q500 (TA Instrument-Waters L.L.C., USA) with a heating rate of $5{ }^{\circ} \mathrm{C} / \mathrm{min}$ under air flow $(50 \mathrm{~mL} / \mathrm{min})$ from $40{ }^{\circ} \mathrm{C}$ to $800{ }^{\circ} \mathrm{C}$.

Depolarized-light intensity measurement: The overall isothermal crystallisation of PLLA was investigated by a GJY-III optical depolarizer with a range of $100{ }^{\circ} \mathrm{C}$ to $120{ }^{\circ} \mathrm{C}$. The electronic signals transformed from the measured optical depolarizer were amplified and then recorded for further analysing.

\section{RESULTS AND DISCUSSION}

\section{Structure of RSX}

The structure of zinc lactate was characterised first using XRD. As shown in Figure 1, the strongest diffraction peak is at $2 \theta=10^{\circ}$, and the diffraction peak position is similar to that in literature (Wang et al., 2011), which indicates that RSX has been successfully prepared. The observations from polarization optical microscopy shows that RSX has an approximate spherical structure (Figure 2). However, literature reports that RSX has a nanowire structure when RSX was prepared using the same

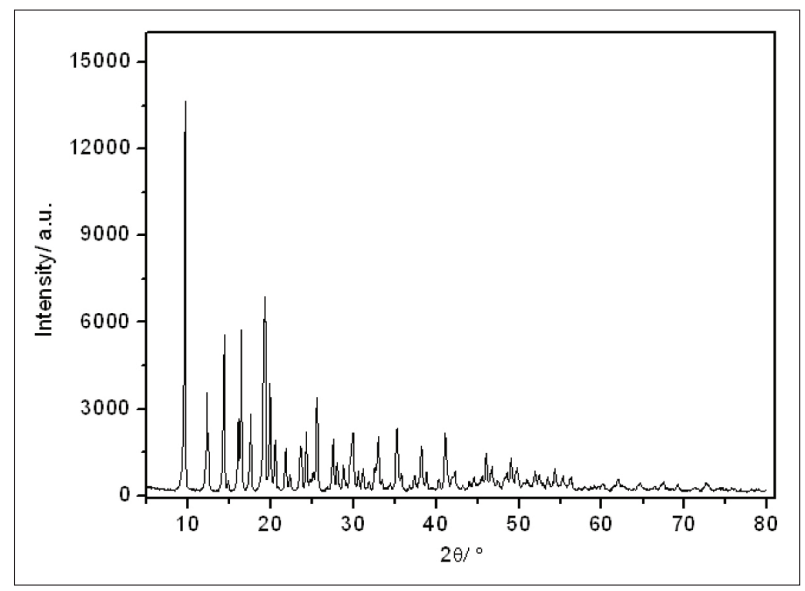

Figure 1: XRD curve of prepared RSX

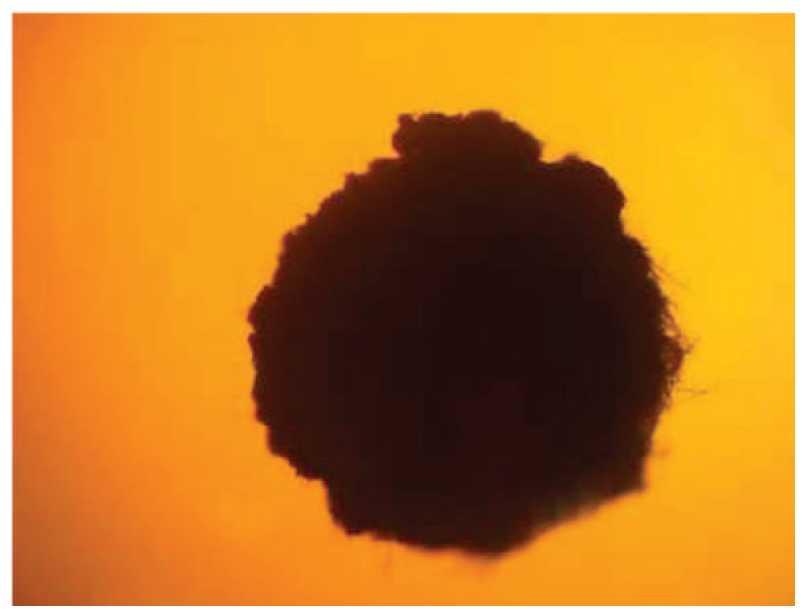

Figure 2: Morphology of RSX observed using microscopy 


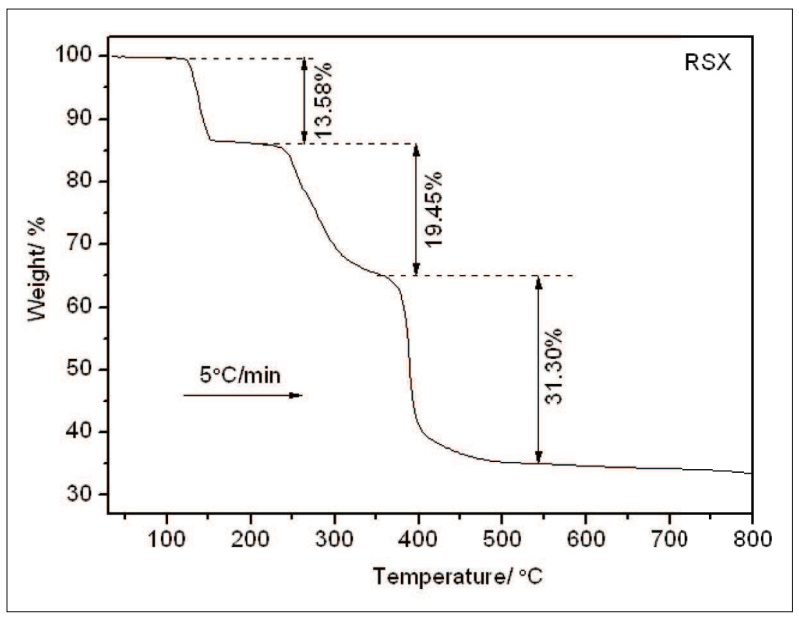

Figure 3: The TGA curve of RSX at a heating rate of $5^{\circ} \mathrm{C} / \mathrm{min}$

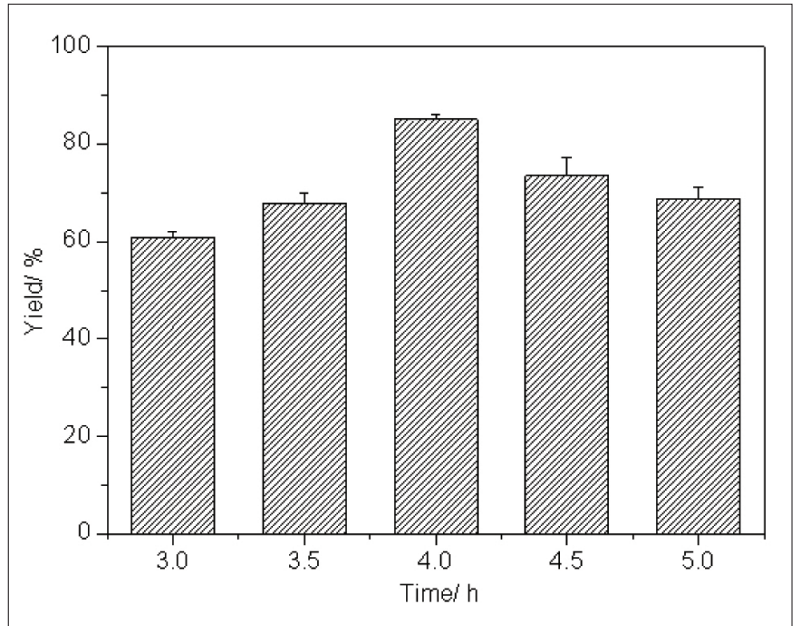

Figure 5: Effect of reaction time on the yield of RSX

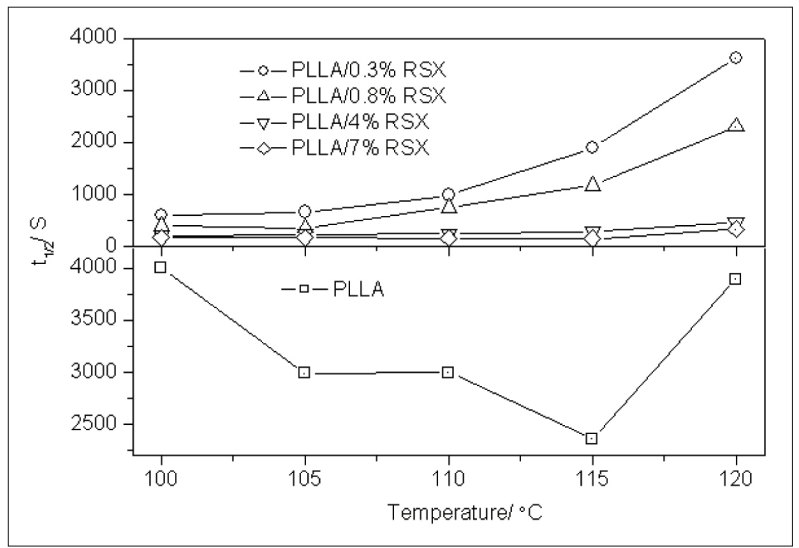

Figure 6: The $t_{1 / 2}$ of PLLA and PLLA/RSX composites with $T_{c}$

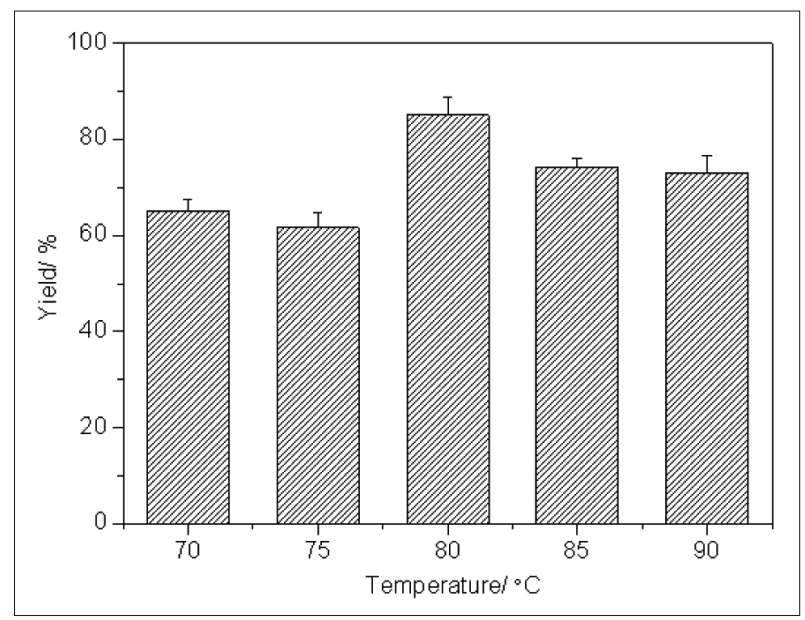

Figure 4: Effect of reaction temperature on yield of RSX

Table 1: Isothermal crystallisation parameters of PLLA and PLLA/RSX

\begin{tabular}{|c|c|c|c|}
\hline Sample & $\mathrm{T}_{\mathrm{c}} /{ }^{\circ} \mathrm{C}$ & $\mathrm{n}$ & $\log K$ \\
\hline \multirow[t]{5}{*}{ PLLA } & 100 & 1.5 & -06.03 \\
\hline & 105 & 2.9 & -10.17 \\
\hline & 110 & 3.3 & -11.69 \\
\hline & 115 & 4.4 & -15.27 \\
\hline & 120 & 5.1 & -20.61 \\
\hline \multirow[t]{5}{*}{ PLLA $/ 0.3 \%$ RSX } & 100 & 4.9 & -13.77 \\
\hline & 105 & 6.2 & -17.89 \\
\hline & 110 & 6.3 & -18.95 \\
\hline & 115 & 5.0 & -16.41 \\
\hline & 120 & 3.3 & -11.91 \\
\hline \multirow[t]{5}{*}{ PLLA $/ 0.8 \%$ RSX } & 100 & 5.3 & -13.97 \\
\hline & 105 & 5.6 & -14.63 \\
\hline & 110 & 6.4 & -18.60 \\
\hline & 115 & 4.4 & -13.55 \\
\hline & 120 & 4.2 & -14.23 \\
\hline \multirow[t]{5}{*}{ PLLA/4 \% RSX } & 100 & 5.2 & -12.17 \\
\hline & 105 & 5.1 & -12.24 \\
\hline & 110 & 5.4 & -13.02 \\
\hline & 115 & 5.2 & -12.93 \\
\hline & 120 & 5.1 & -13.97 \\
\hline \multirow[t]{5}{*}{ PLLA/7 \% RSX } & 100 & 6.2 & -14.19 \\
\hline & 105 & 5.5 & -12.33 \\
\hline & 110 & 5.3 & -11.90 \\
\hline & 115 & 4.9 & -10.80 \\
\hline & 120 & 4.2 & -10.88 \\
\hline
\end{tabular}

synthesis method (Song et al., 2012), depending upon the stirring modes and stirring rate. Compared to normal RSX, RSX with high specific surface area has more advanced nucleation ability for PLLA (Song et al., 2012). 
RSX with a spherical structure may significantly enhance the crystallisation properties of PLLA.

Thermal studies of RSX were carried out using TGA. The TGA curve of RSX at a heating rate of $5{ }^{\circ} \mathrm{C} / \mathrm{min}$ is presented in Figure 3. It is clear that there exists three platforms on the TGA curve(Figure 3). The decomposition temperature of the first stage is $125{ }^{\circ} \mathrm{C}$, resulting from the loss of absorbed water, corresponding to a mass loss of $13.58 \%$. The decomposition temperature of the second stage is $257^{\circ} \mathrm{C}$, and the mass loss is $19.45 \%$. The decomposition temperature of RSX is higher than the

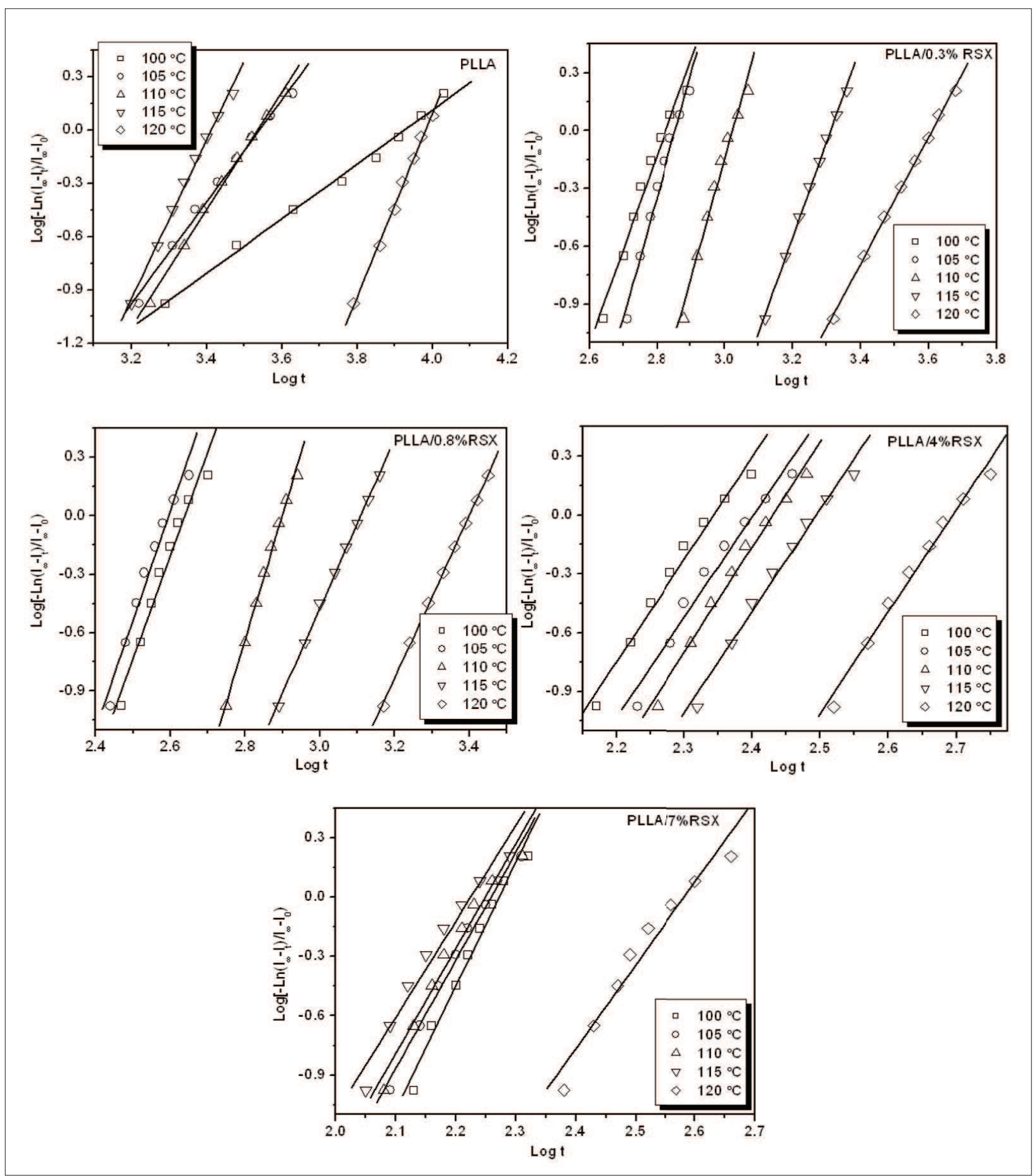

Figure 7: Avrami plots for PLLA and PLLA/RSX at different temperatures 
processing temperature of composites based on PLLA, which indicates that RSX can serve as a nucleating agent for PLLA. The maximum mass loss is in the third stage, which is $31.30 \%$.

\section{Synthesis process of RSX}

The influence of reaction temperature and reaction time on the yield of RSX was investigated using single factor experiments. First, using a solution with $5 \mathrm{~mL}$ of water and $2.5 \mathrm{~mL}$ of L-lactic acid, the effect of reaction temperature on the yield of RSX was evaluated for a 4 hour reaction time. The experimental results are shown in Figure 4. The yield of RSX increased with increasing reaction temperature. At the reaction temperature of $80{ }^{\circ} \mathrm{C}$, the yield of RSX has a maximum value of $84.97 \%$. Appropriately increasing the reaction temperature can improve reaction activity, but higher reaction temperature will also cause the increase of byproducts, resulting in decreased yield.

Using a solution with $5 \mathrm{~mL}$ of water and $2.5 \mathrm{~mL}$ of L-lactic acid, the influence of reaction time to the yield of RSX was investigated at an optimum reaction temperature of $80{ }^{\circ} \mathrm{C}$. The effect of reaction time on the yield of RSX had a similar trend with that of reaction temperature (Figure 5), and the maximum yield occurs with the reaction time of 4 hours. Thus, the optimum reaction conditions are: reaction temperature of $80{ }^{\circ} \mathrm{C}$ and reaction time of 4 hours.

\section{Isothermal crystallisation of PLLA/RSX composites}

According to the results of morphological study and thermogravimetric analyses, RSX as nucleating agent may improve the crystallisation of PLLA. Thus, we investigated the isothermal crystallisation behaviour of PLLA/RSX composites. Figure 6 shows the isothermal crystallisation behaviour of PLLA/RSX composites. It is clear that the half-time of composite PLLA crystallisation $t_{1 / 2}$ becomes low after the addition of RSX. Furthermore, with the increase of RSX content, $t_{1 / 2}$ becomes lower, which further indicates that RSX can improve the crystallisation of PLLA, and increase the crystallisation rate of PLLA. A high content RSX significantly reduces the half-time of composite PLLAcrystallisation. However, the change in the half-time of PLLA crystallisation is not regular with the increase of crystallisation temperature, showing that PLLA/RSX composites have a complicated crystallisation process. Compared to pure PLLA, the half-time of composite PLLA crystallisation $t_{1 / 2}$ decreases from 2359.91 seconds to 152.68 seconds at $115{ }^{\circ} \mathrm{C}$ with the addition of $7 \%$ RSX.
Avrami equation can be used to describe the kinetics of isothermal crystallisation of PLLA/RSX composites. The measure and theoretical basis for isothermal crystallisation have been described previously (Cai et al., 2012). Figure 7 shows the Avrami plots of PLLA and PLLA/RSX composites. The relationship of $\log \left[-\ln \left(1-\mathrm{X}_{\mathrm{t}}\right)\right]$ versus $\log \mathrm{t}$ is linear. $\mathrm{X}_{\mathrm{t}}$ represents the percentage of relative crystallisation after time $t$ in the measure method. The kinetic parameters $n$ and $k$ values of pure PLLA and PLLA/RSX composites are listed in Table 1. Usually, $n$ is four (4) for a homogeneous nucleation system and three (3) for a heterogeneous nucleation system. However, Table 1 shows that the $n$ values of pure PLLA is in the range of $1.5-5.1$, and the $n$ values of PLLA/RSX composites range between 3.3 - 6.4. These results show that the crystallisation process of PLLA and composites based on PLLA are very complicated, and may be affected by RSX, crystallisation temperature, operation process, etc. Furthermore, a similar phenomenon has been reported in other studies (Li et al., 2009). But the variation of $k$ values has a similar trend as that of $t_{1 / 2}$ values.

\section{CONCLUSION}

Spherical RSX with a high specific surface area was synthesized from zinc acetate and L-lactic acid. Thermal decomposition showed a higher decomposition temperature of RSX. The optimum reaction conditions of RSX were a reaction temperature of $80{ }^{\circ} \mathrm{C}$ and a reaction time of 4 hours. Under these optimum conditions, the yield of RSX was $84.97 \%$. Isothermal crystallisation behaviour indicated that RSX could significantly decrease the half-time of composite PLLA crystallisation. Compared to pure PLLA, the half-time of composite PLLA with 7 \% RSX decreased from 2359.91 seconds to 152.68 seconds at $115{ }^{\circ} \mathrm{C}$.

\section{Acknowledgement}

This work was supported by the National Natural Science Foundation of China (Project No. 51403027), China Postdoctoral Science Foundation (Project No. 2013M531937), Natural Science Foundation Project of CQ CSTC (Project No. cstc2012jjA50001), Scientific and Technological Research Programme of Chongqing Municipal Education Commission (Project No. KJ131202) and Chongqing University of Arts and Sciences (Project number R2012CH10, 2012PYXM04 and Z2011CL11). 


\section{REFERENCES}

1. Cai Y.H., Wang F., Zhang Q., Hao H.T., Zhang Y.H., Tian Y.Y. \& Li S.J. (2012). Isothermal crystallisation behaviour and mechanical properties of poly (L-lactic acid)/Talc/EBS composites. Asian Journal of Chemistry 24: 4949 - 4952.

2. Cai Y.H. (2013). Synthesis of $N, N$ '-bis(benzoyl) azelaic acid dihydrazide and its effect on performance of poly(L-lactic acid). Journal of the Chemical Society of Pakistan 35: 1174 -1178 .

3. Cai Y.H., Fan Y.Q. \& Tian Y.Y. (2014). The nucleation effect and thermal behaviour of poly (L-lactic acid) in the presence of a bisamides compound. Journal of the Chemical Society of Pakistan 36: 371 - 376.

4. Campardelli R., Della Porta G., Gomez L., Irusta S., Reverchon E. \& Santamaria J. (2014). Au-PLA nanocomposites for photothermally controlled drug delivery. Journal of Materials Chemistry B 2: 409 - 417. DOI: http://dx.doi.org/10.1039/c3tb21099e

5. Cao X.J., Lee H.J., Yun H.S. \& Koo Y.M. (2002). Crystallisation and dissolution behaviour of $\mathrm{L}(+)$ calcium and zinc lactate in ethanol-water solvent. Korean Journal of Chemical Engineering 19: 301 - 304. DOI: http://dx.doi.org/10.1007/BF02698418

6. Jo M.Y., Ryu Y.J., Ko J.H. \& Yoon J.S. (2012). Effects of compatibilizers on the mechanical properties of ABS/PLA composites. Journal of Applied Polymer Science 125: E231 - E238.

DOI: http://dx.doi.org/10.1002/app.36732

7. Katiyar V., Gerds N., Koch C.B., Risbo J., Hansen H.C.B. \& Plackett D. (2011). Melt processing of poly(L-lactic acid) in the presence of organomodified anionic or cationic clays. Journal of Applied Polymer Science 122: 112 - 125.
DOI: http://dx.doi.org/10.1002/app.33984

8. Li X.X., Yin J.B., Yu Z.Y., Yan S.F., Lu X.C., Wang Y.J., Cao B. \& Chen X.S. (2009). Isothermal crystallisation behaviour of poly(L-lactic acid)/ organo- montmorillonite nanocomposites. Polymer Composites 30: 1338 - 1344. DOI: http://dx.doi.org/10.1002/pc.20721

9. Longano D. et al. (10 authors) (2012). Analytical characterization of laser-generated copper nanoparticles for antibacterial composite food packaging. Analytical and Bioanalytical Chemistry 403: 1179 - 1186.

DOI: http://dx.doi.org/10.1007/s00216-011-5689-5

10. Song P., Chen G.Y., Wei Z.Y., Wang D.J., Liang J.C. \& Zhang W.I. (2012). Effect of zinc lactate nano-wires as uncleating agent on the crystallisation of poly (lactic acid). Journal of Functional Materials 43: $42-45$.

11. Tang Z.B., Zhang C.Z., Liu X.Q. \& Zhu J. (2012). The crystallisation behaviour and mechanical properties of polylactic acid in the presence of a crystal nucleating agent. Journal of Applied Polymer Science 125: 1108 - 1115. DOI: http://dx.doi.org/10.1002/app.34799

12. Wang D.J. (2011). Effects of several nano-materials on the crystallisation and mechanical properties of poly (lactic acid). Dalian University of Technology: 21

13. Zhang X.Y., Fevotte G., Zhong L., Qian G., Zhou X.G. \& Yuan W.K. (2010). Crystallisation of zinc lactate in presence of malic acid. Journal of Crystal Growth 312: $2747-2755$.

DOI: http://dx.doi.org/10.1016/j.jcrysgro.2010.06.005

14. Zhang X.Y., Qian G. \& Zhou X.G. (2012). Effects of different organic acids on solubility and metastable zone width of zinc lactate. Journal of Chemical and Engineering Data 57: 2963 - 2970.

DOI: http://dx.doi.org/10.1021/je3006453 